\title{
Shape Analysis and Visualization in Building Floor Plans
}

\author{
Katharina Roth*, Eva Hagen ${ }^{\dagger}$ and Christina Gillmann ${ }^{\ddagger}$ \\ ${ }^{*}$ Computer Graphics and HCI Group, University of Kaiserslautern, Kaiserslautern, Germany \\ E-mail: kroth@rhrk.uni-kl.de \\ ${ }^{\dagger}$ Darstellende Geometrie und Perspektive, University of Kaiserslautern, Kaiserslautern, Germany \\ E-mail: eva.hagen@architektur.uni-kl.de \\ ${ }^{\ddagger}$ Signal and Image Processing Group, Leipzig University, Leipzig, Germany \\ E-mail: gillmann@informatik.uni-leipzig.de
}

\begin{abstract}
Building floor plans are an important tool for architects to derive new designs or analyze given designs. Here, architects are interested in the analysis of existing designs. Unfortunately, building floor plans need to be analyzed by architects manually, which requires a lot of time. This paper builds on a close collaboration between computer scientists and architects. The provided analytical methods allow extracting geometric primitives in building floor plans, resembles them to superior structures, and analyze their location and number of occurrences in a building floor plan. In this work, we show the effectiveness of the presented approach by applying it to a realworld building floor plan and discuss the results.

Index Terms-Shape Detection, Architecture, Geometry Visu-
\end{abstract} alization

\section{INTRODUCTION}

Architects always search for novel ideas on how to design a new building. Here, the usual approach of an architect is to look at already existing works or building plans and examine them concerning their relevant characteristics. Such characteristics can be, for example, the purpose of the building to be constructed or requirements like budget, environmental restrictions, or legal restrictions. However, the most important characteristic for architects in this context in terms of aesthetics is the analysis of the geometric shapes contained in a building.

Starting with the pyramids in Egypt thousands of years ago, today, various geometric components are brought together to build a new structure. Geometry is a concept not exclusive to monuments and famous buildings, but it belongs to all constructions. Architectures can be described geometrically. They consist of basic geometric patterns from formal order systems that come into play in the design process.

Therefore, the analysis of floor plans on geometric principles like basics forms, transformations, golden ratio, mirroring, and proportion can yield valuable insight into the system behind a structure. Its functionality or comfort for the user will be expressed in its layout using various features [1].

The identification of correlations between different features of floor plans is therefore a major goal for architects. Based

LEVIA'20: Leipzig Symposium on Visualization in Applications 2020 on this analysis they can use these insights to create new designs, fusing together several layouts into one structure with properties from both worlds.

The analysis of the existing floor plans for room geometries gives the architect the opportunity to break down comparable projects to their room elements during the design process. This allows conclusions to be drawn about the spatial effects, the uses of the space and shows the relationship between the function and size of the rooms. Furthermore, the analysis tool could be used to type buildings according to geometrical criteria in order to find the perfect typology for your own project.

This type of analysis is currently performed manually by architects, which requires an enormous amount of time and effort. With this initial problem, our collaboration partners from the field of architecture approached us to develop a tool that recognizes geometric figures in a floor plan to facilitate the analysis.

As mentioned above, this work is based on a close collaboration between visualization researchers and researchers from the architecture domain. We provide a workflow to identify all geometric objects used in a floor plan and characterize their properties. These properties are analyzed to extract their proportions, angles, and distances, creating a specific fingerprint for the floor plan. Then the insight from various floor plans can be used to categorize those structures into distinct classes, while including information like the architect, the age, the use case, used materials, or location of the building. Similar buildings concerning their geometric properties can be found and the architect can locate similar layouts to use in their design, as shown in Section IV. Resulting from this, architects can filter designs and find inspiration for new projects.

Therefore, this paper contributes:

1) A tool to identify geometric forms in floor plans

2) Pitfalls when analyzing geometric forms

3) An outlook on how to use the analysis of floor plans for further applications

We show the applicability of the presented approach by analyzing a real world data set provided by our application 


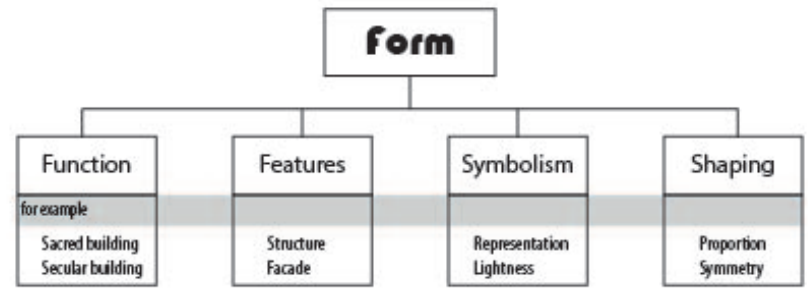

Fig. 1. Form finding process in Architecture. This process can be divided into function, features, symbolism and shaping.

collaborators and discuss the advantages and limitations of this approach in Section V.

\section{ApPLiCATION ANALYSiS}

In this section we aim to provide further details about the presented application driven problem by defining the problem itself and explaining the manner of the available data sets. Based on this analysis we are able to provide a requirement analysis for the presented problem.

\section{A. Problem Description}

A crucial point in this respect is that each architect has an individual form of analysis. Each architect attaches different importance to different aspects of the design of a new building. This was expressed during our cooperation. When asked where and which geometric shapes are to be seen in a given floor plan, the architects interviewed gave very different information. For example, a room with rounded corners was perceived by some as a rectangle, by others as a rectangle with four circles.

Furthermore, during our work, we have experienced that an architect rejects all kinds of tools that in any way limit his 'artistic freedom'. A decisive criterion for the acceptance of our tool is to offer the user many design options to represent his ideas within our tool.

An architect spends a lot of time with research. For example, plans of buildings with a similar purpose are compared with buildings that have a design style similar to the architect's ideas. The number of buildings to be compared is sometimes very high.

The analysis of building design is a complex field, as shown in figure 1. It consists of function, feature, symbolism, and shape analysis. In the presented case, solely building floor plans are available as input data sets. Resulting from this, solely shape analysis can be applied.

Based on this problem, architects are aiming for a system that allows them to understand the used shapes in a building, their frequency, and their composition. Here, the idea is to derive rules for aesthetic building designs.

This forms the motivation of the presented work. In this work, we aim to analyze the shapes used in building floor plans. At this point, we want to clarify, that we provide an overview of the used shapes and their frequency, but the derivation of rules will be performed by the architects.

\section{B. Data}

The data was provided by our collaboration partners utilizes the Windows bitmap format to allow easy reading. These images show illustrations of real buildings to ignore smaller details that might overload the image as shown in figure 2 . This means that the plans do not contain any additional information, such as wall thickness or room size. Also, no windows and doors are shown in the plans utilized plans. In the current stage of the project, the architects are solely interested in the utilized shapes in order to understand patterns in building design. This does not include windows, doors, or other facilities. Rather, the floor plans represent some kind of skeleton of the individual rooms and structures, for a better analysis. For real floor plans, simple changes could be made to suffice to the standards we utilize.

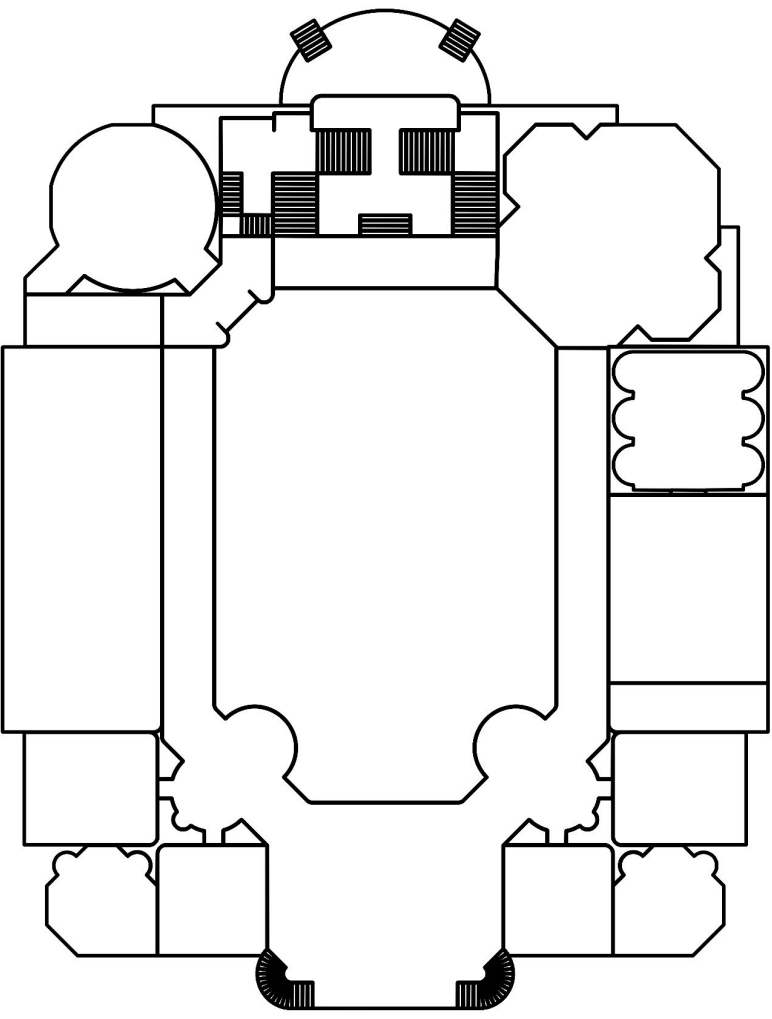

Fig. 2. Example of a floor plan, which we receive as input data

\section{Requirements}

In order to provide a sufficient analysis of building floor plans, several requirements have to be fulfilled. These requirements will be discussed in the following. They are formulated based on long discussion with our collaborators from architecture. 
1) Level of Detail: To satisfy the needs of various architects, the level of detail for the geometry analysis has to be considered. Some architects only focus on the outside of the building while others need very detailed information about each room they consider using. Therefor the analysis of floor plans has to be conducted in several levels of detail [2].

1) global outer shape:

Detection and analysis of the outside of the building

2) local outer shape:

Detection and analysis of the inside of a building

3) global inner shape:

Detection and analysis of the outside of the room

4) local inner shape:

Detection and analysis of the inside of each room

Each of theses classes focuses on either the inside or outside of buildings or rooms. The global outer shape is distinct from the other classes, but the local outer shape is a union of the remainders.

2) Connectivity analysis: The analysis of rooms can be conducted in two different ways. First, the connectivity information between rooms can be omitted. This analyzes each room on its own, while not considering its neighborhood information. This can lead to performance gains as each room is being analyzed once and calculations can be carried out in parallel. But, in rooms with nested structures, additional calculations still have to be conducted. Another way to analyze the layout of a building is to use the connectivity between rooms, s.t. the neighborhood information of each room and wall is used. Each segment of a room saves the adjoined segment of the opposite room. Using this method not only gets rid of redundant calculations but also grants valuable insight into the layout of the building.

\section{RELATED WORK}

In this section we aim to discuss related work to our approach in terms of shape detection applications and their application to architecture.

\section{A. Shape Detection}

Besides floor plan analysis, shape detection is an often required processing step in many applications, such as face detection [3], text detection [4], bar code detection [5], building detection [6] or medical structure detection [7].

Shape detection algorithms can be roughly differentiated into analytical approaches and machine learning approaches. Analytical approaches aim to analyze images based on identifying geometric primitives mathematically and assemble them to different shapes. Machine learning algorithms on the other hand aim to learn different shapes based on a training database.

There exists a variety of analytical shape detection approaches, as shown by Loncaric et al. [8]. In his work, he summarized analytical approaches for shape detection in images. Although this work summarizes existing shape detection approaches, the selection of proper shape detection algorithms is highly dependent on the underlying data. Here, proper algorithms need to be chosen to address the task that needs to be fulfilled.

Recently, deep learning approaches became quite popular in the field of shape detection [9]. Although this technique output promising result and often outperform analytical approaches, they require a large training database. In the given project, this database was not available. Resulting from this, our approach relies on analytical methods that are wisely chosen to fulfill the needs of our collaborators.

\section{B. Shape Detection in Architecture}

In architecture, shape detection is an important task [10]. It ranges from the analysis of building photography [11], [12] to building floor plan analysis [13]. In this work, we will focus on building floor plan shape analysis, as this was the formulated task by our collaborators in architecture.

Dodge et al. [14] presented a computer vision approach that aims to identify walls in a building floor plan to automatically create a three-dimensional model of the building. The work utilizes a wall segmentation approach to extract the structures that need to be model in 3D. Unfortunately, this approach does not provide an analysis of the extraction rooms and walls in terms of their shape. In contrast to this, our approach classifies the extracted structures and computed the geometric shape.

Gillmann et al. [15] presented a visual system to allow the application of an arbitrary image processing algorithm and review their output. Although this allows users to understand the applied algorithms, it cannot be utilized in the current scenario as the architects aim for an automatic system. Here, we present an automatic analytical system with well-selected algorithms that allow architects to analyze building floor plans.

Mace et al. [16] provided an algorithm that partitions the identified interior of a building in a floor plan into different rooms. Although their approach outputs accurate results, they do not provide an analysis of the resulting shapes of the rooms. Therefore, the presented approach also provides a shape analysis of the extracted rooms.

Okorn et al. [17] provided a tool to automatically model floor plans based on points clouds that are captured from a building. Here, floor plans have been created that show the different walls in a building. Unfortunately, the shape of the rooms is not analyzed. Therefore, this work presents a shape analysis of building floor plans, that could also be applied to the work of Okorn et al.

Jang et al. [18] presented a wall detection algorithm that detects different walls in building floor plans and analyzes their geometry. The result is a graph representing the walls and where they are connected. Still, the method does not analyze the resulting shapes of different rooms. We will use this work as a starting point and add the component of shape analysis to it.

Ziran et al. [19] presented an algorithm for object detection in building floor plans. In their work, they focus on identifying objects that are located in a building floor plan a label them. Although this approach includes shape analysis to achieve proper labeling of objects, the shapes are not communicated 
to the user. In our approach, we derive a shape analysis and provide our medical collaborators the results of this analysis.

In general, the presented approaches all work on straight shapes. This means, that no curved objects or walls were examined. In architecture rounded walls are quite popular, resulting in the need for a shape analysis for these structures as well. In this work, we include the analysis of such structures as well.

\section{METHODOLOGY}

In this section, we present the developed methodology that performs automated shape analysis on building floor plans. The methodology consists of four steps, that are shown in figure 3 .

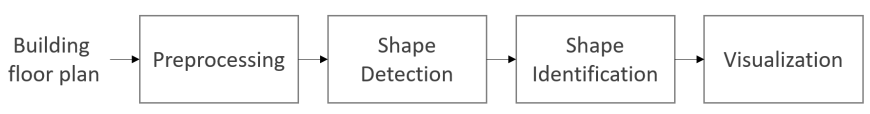

Fig. 3. Overview of the presented approach. Strting from a building floor plan, the steps preprocessing, shape detection, shape identifcation and visualization are performed.

This pipeline will be described in detail in the following. The components are implemented using the framework OpenCV [20] and was programmed in $\mathrm{C}++$.

\section{A. Preprocessing}

Before we can carry out an analysis of the individual rooms, they must be segmented first. To do this, we determine an edge image of the input floor plan using the canny-edge detection algorithm and then identifying the pixels that belong to the building or the individual walls using the Probalistic Hough line method [21].

After extracting the background of the image, the image is now inverted in such a manner that the area of the individual rooms is clearly separated from one another. Based on this step, the shape of the individual rooms can be determined in the following steps.

\section{B. Shape detection}

After the segmentation of the individual rooms, we aim to apply shape detection algorithms in order to determine the shapes of the individual rooms. For further analysis it is necessary to determine individual segments that represent characteristic parts of the area that assembles an individual room. In the case of a straight wall, the segment represents the whole wall. However, in case of a curved wall these segments need to be divided into several subsegments.

To achieve this, the shape recognition step is divided the following three parts.

1) Contour recognition

2) Segmentation of the characteristic room sections

3) Classification of the characteristic room sections

Each step shown in figure 4 will be explained in the following.
1) Contour recognition: To solve this problem we decided to use of topological structure analysis [22] already implemented in OpenCV.

The algorithm has two different modes:

1) with approximation of the shape (solely corner points of the room will be extracted)

2) without approximation of the shape (all pixels that are located on the border of the room will be extracted)

Both modes reveal important information for different stages in the presented shape analysis algorithm, so both algorithms were applied to the image. As a result of that, we get two sorted list of points that describe the contour of the respective room. One with all points of the contour and one with an approximated set of points.

2) Segmentation of the characteristic room sections: After we recognized the contour of the entire shape we focus on the division of the individual room boundaries into meaningful sub-segments. Therefore, we define that approximated points of a shape describe the beginning and end of a segment and that all points between two successive approximated points belong to one segment.

3) Classification of the characteristic room sections: For the identification of the shape it is necessary to know in advance whether a segment is a curved or a straight fragment of a room. For this reason a classification into these two categories is necessary. This separation into those two categories can be achieved by using the hough line algorithm [21]. This algorithm is able to identify straight lines in an image. Although the line representation needs to be derived further, the identified segments from the hough transformation form a suitable starting point.

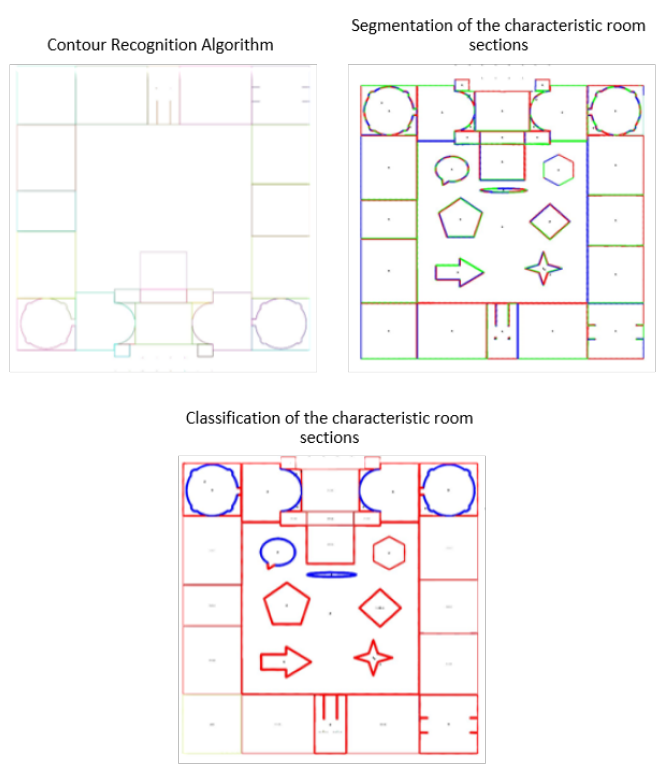

Fig. 4. Example of the result after shape detection 


\section{Shape identification}

Since we achieved a description of the contour of each room, we will address the problem of shape identification. Shape identification is the definition of the geometric shapes that make up a contour. We differentiate between so-called primitives also called simple basic shapes, such as rectangles or circles, and complex shapes. There are different types of complex figures that behave analogously to the types that can be found in set theory, such as union or intersection.

There can be several ways in which a given contour can be categorized. In this context, the question arises which of the numerous possibilities an architect sees as the "correct one". Therefore, the number of shape classes has to be reduced and the priority rules for those shapes need to be very exact.

As explained above, many complex forms can be described using primitives. In addition, there exist primitives that can be described by other primitives. For example, a rectangle can also be represented using two triangles. From a computer graphics view, any shape can be described by an arbitrary amount of triangles with a certain degree of accuracy, but the benefits for the architect would be negligible in this case. In addition, a representation that is solely composed of triangles does not correlate with the intuition of architects when reviewing different shapes.

In cooperation with our collaboration partners, the following classification of primitive classes was created: square, rectangle, triangle, ellipse, circle, polygon, curved shape

Note that the classes Polygon and Curved Shape are Weak Primitive Classes. Weak Primitive Classes are necessary, because there are more Primitive Classes than the ones we have chosen for this application. All other Primitives are represented by the Weak Primitive Classes. Any complex shape that consists exclusively of straight lines is a polygon in the mathematical sense. In our case, the primitive class Polygon describes all complex shapes that consist exclusively of straight lines and cannot be assigned to any other primitive class. I.e. a square is not part of the primitive class Polygon. Furthermore, the class Curvy Shape includes all curved shapes that are based on a variety of curvature changes. This class was introduced primarily for reasons of clarity.

Shape detection provides us with the segments of a contour including information about the type of segments, i.e. we know from each segment of the contour whether it is a straight or a curved line. To identify a given primitive/complex shape, the number of segments marked as curves and the number of segments marked as straight lines has to be determined. Thus, a separate analysis can be performed with respect to the occurrences of representations from primitive classes of curved shapes and straight shapes.

For the evaluation of each line segment, its representation is interpreted as a vector defined by its endpoints. In the following, the recognition methods of straight and curved geometric shapes are explained in more detail.

1) Recognition of rectilinear geometric shapes: For the identification of straight geometric shapes, the relationship between straight and curved segments is being considered.
If the number of curved segments is zero, we can use the information about the number of segments to speed up the classification. For example, in this case, we know that if we find more than four segments, it is definitely a representative of the Weak Primitive Class polygon.

If a complex shape is present, i.e. the number of curved segments is not equal to zero, we can not or only partially include the number of straight segments in the identification, as mentioned above. This is due to the fact that linear segments do not necessarily follow each other directly and thus they may not be related to each other at all or in a different relationship as assumed in the primitive analysis. An example of this would be if two straight line segments are detected, but they are parallel to each other. In this case, it is either two single lines or even a partial rectangle or a partial square.

The following section describes how the linear forms are classified.

First, we will focus on rectangles. One of the main characteristics of a rectangle are two pairs of parallel lines of equal length. For this reason we look for all possible pairs of straight segments of a shape that are parallel to each other. This is done by vector calculus. Vectors are also considered parallel if they are identical, which means that segments lying on the same straight line are recognized as rectangles. This problem is solved by additionally checking whether the vector defined by the two starting points of the segments is also parallel to the two segments.

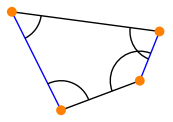

(a)

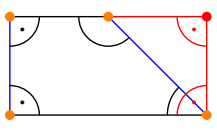

(c)

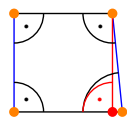

(e)

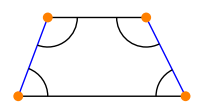

(b)

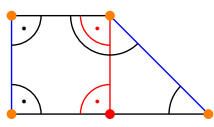

(d)

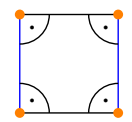

(f)
Fig. 5. Cases of Quadrangles: a) segments are not parallel and have no right angles, b) segments are parallel and have no right angles, c) Large projection, d) Small projection, e) Noise correction, f) Base case

After a set of pairs of parallel segments has been determined, we use the property of a rectangle that has exactly four right angles. We form two connecting imaginary lines by connecting the corner points of a pair of segments such that a rectangle is formed. It is not important whether these imaginary lines really exists. Then we determine all four angles that have been created and check whether they are right angles. Now, all four angles that have been calculated and need to be checked whether they are right angles. 
Since imaginary rectangles need to be recognized, i.e. those which only have an indicated form, a rectangle needs to be identified if at least two or at most four right angles have been calculated.

This results in the following cases which are illustrated in figure 5:

1) Parallel Lines of Unequal Length:

In this case, the user decides between two display modes: Large and Small Projection.

a) Small Projection: If two right angles are found on an imaginary line, a valid rectangle has been found and a second imaginary line can be build by projecting the shorter line onto the longer one. The remaining triangle can also be saved for later analysis.

b) Big Projection: In contrast to the Small Projection, the small segment is now adjusted to the size of the larger one, creating a rectangle.

2) Noise:

noisy data can result in inaccuracies that lead to the fact that a perfect rectangle no longer exists. These deviations have to be taken into account in the rectangle detection.

3) parallel line of equal length:

This is the simplest and clearest case, since it is clearly a rectangle.

Since we reconstruct a rectangle from two parallel lines, we may recognize a rectangle more than once if real images of the connecting lines exist in the shape.

Once we have identified a rectangle, we check whether it is a square. Therefore, we check whether the connecting lines are the same length as the two segments. If it is a projected rectangle, the newly calculated connection line is used for the determination.

After all rectangles have been identified, we determine how many rectilinear segments have not yet been assigned to a primitive class. Depending on how many unclassified segments follow each other, i.e. form a stretch, we assign them to primitive classes. This means that if, for example, there are three contiguous straight-line segments that do not form a rectangle, they are combined into a triangle. If there are more than three consecutive segments that do not form a rectangle, it is a polygon.

2) Recognition of Curved Geometric Shapes: Since the circle is a special case of an ellipse, we first concentrate on the detection of the ellipse and then check whether the found ellipse is a circle.

We can determine which segments have a curved course, but not whether it is one, several, or no ellipse at all. If the spatial shape has several ellipses, there is the additional problem of distinguishing which segment belongs to which ellipse. An analogous problem arises with a set of curvatures that are not ellipses. So our first task is to define stretches of segments that clearly belong to the same ellipse.

With the help of the angle condition described in the work of Nguyen et al., prominent buckling in the course of the curve can be detected, from which it can be concluded that all segments between these points (keypoints) will most likely belong to the same ellipse [23]. We call these sets of segments ellipse parts. The success of the calculation of these ellipse parts depends strongly on the number of points, i.e. the sampling theorem plays a decisive role here.

This procedure only recognizes which directly adjacent segments belong together. To detect even those parts of the ellipse that are not directly related a method for grouping ellipse parts is described below, which was introduced by Prasad et al. [24].

First, the tangents of the start and endpoints must be calculated for all ellipse parts. The area spanned by the two tangents, in which no point of the currently considered section of the ellipse is located, defines the so-called search region. An ellipse part is recognized as a possible part of the same ellipse if it is contained in the search region of the currently viewed ellipse part and vice versa. Here we define that an ellipse part is included in a search region if all its points are located in the search region. As a result, we get a set of possible valid ellipse parts. Figure 6 illustrates this connection.

In this example, $e_{2}$ and $e_{6}$ are recognized as invalid sections as they are outside the search region. $e_{3}$ is also an invalid option because the search region of this elliptical part does not include all points of the currently viewed ellipse part. This means that $e_{4}, e_{5}$, and $e_{7}$ are potentially valid parts of the same ellipse.

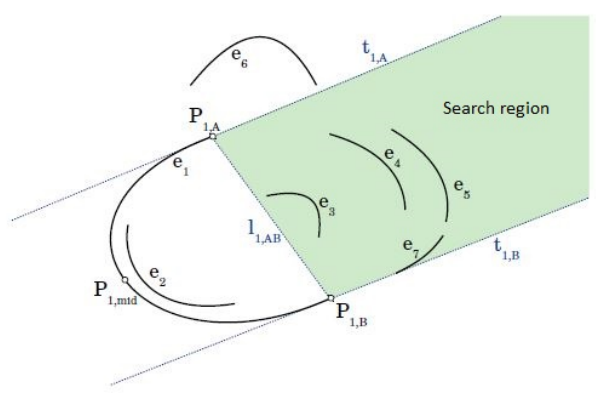

Fig. 6. Definition of a search region of ellipse parts for a possible grouping [24]

After creating for each ellipse part a corresponding set of possible parts of the same ellipse, these sets must be compared to decide which parts belong together.

Finally, we apply the fitEllipse() algorithm implemented in OpenCV, based on the method of Fitzgibbon et al., to reconstruct the ellipse [25].

To decide whether it is a circle or not, we check whether the resulting bounding box is a square.

\section{Visualization}

In order to present the result of the performed shape analysis to our collaborators, we provide a visualization that summarizes the extracted shapes, their location, and their distribution. Our visualization consists of two components. First, we provide a view of the original floor plan including 
the identified shapes. In addition, we provide a bar chart that summarizes the number of different shapes in the building floor plan. We aimed for an intuitive design [26] that will be accepted by our architectural collaborators. An example visualization for a small example can be seen in figure 7 .

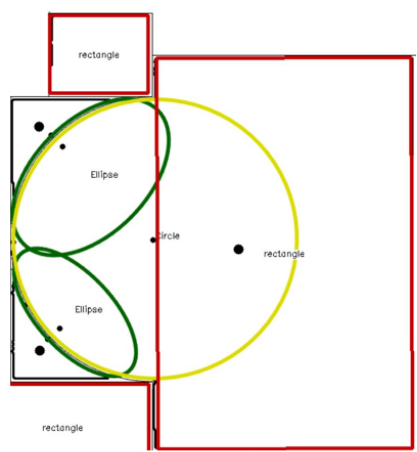

a)

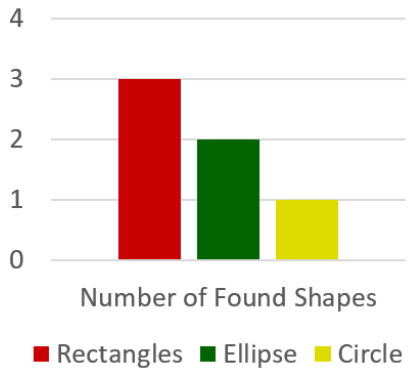

b)
Fig. 7. Visualization of shape analysis result. a) Building floor plan with identified shapes as overlay. b) Barchart with number of found objects per shape.

Figure 7 shows the results of the shape analysis for a small example. The original floor plan view is provided in a). Here the original image of the floor plan is shown. As an overlay, the identified shapes (rectangle, ellipse, and circle) are shown in distinct colors as an overlay. Each of the drawn shapes is labeled by the respective class name that was identified. In case, an identified shape solely shows a part of the shape, the entire shape is drawn to show the user where the shape would be located if it was complete.

Figure $7 \mathrm{~b}$ ) shows a bar chart capturing the number of found shapes in the floor plan. In this case, 3 rectangles, 2 ellipses, and one circle. The color of each bar corresponds to the drawn shapes in the floor plan view.

These two views allow architects to identify which shapes are present in the given floor plan and shows a comparative view of their frequency.

This example shows five different spaces or recognized contours and the respective result of our algorithm. Due to the fact that recognized contours are analyzed individually, two ellipses are created in this example, which is to be regarded as errors in our context. This kind of error can be detected by a superordinate global analysis of the contours, which we will deal with in future work.

\section{RESUlts AND DISCUSSION}

In this section, we will present the application of the provided shape analysis and visualization approach to a real world floor plan. In addition, we discuss the benefits and drawbacks of the presented approach.

\section{A. Results}

To show the effectiveness of our approach, we applied the presented methodology to a real-world floor plan. We considered the example of the Glyptothek in Munich [28].
The building holds a museum of antique arts and is a popular example of classicism architecture.

The resulting visualization can be reviewed in figure 8 . Our method was able to identify 5 different shapes in the building floor plan: rectangles, circles, ellipses, squares, and triangles. it can be observed, that the building mostly consists of rooms of rectangular shape. The two squared rooms are located in the two upper corners. In the lower part of the image (entrance of the building), several shapes can be found. Here, rooms that are composed of rectangular and round shapes can be decomposed into their compartments. For example, in the lower-left corner a round room with round embayment can be decomposed into the big circle and the smaller circles that form the embayment.

We showed this example to our collaborators from architecture, and they were very satisfied with these results. In comparison to their standard working routine, they are now able to identify different shapes and research their proportions and compositions faster. Basic geometric shapes always form the basis of every building design, so the current status of our tool also forms the basis for the subsequent analysis steps to find geometric principles such as the golden ratio.

For testing our tool, hand-labeled plans were compared with the results of our tool. Even though the results were not always perfect, our collaboration partners considered the overall results a success. Most of the discrepancies between the hand-labeled data and the results of our program are due to the lack of global analysis, as mentioned above, which would relate the individual rooms to each other. Another very common source of error is data that was too noisy.

\section{B. Discussion}

The presented algorithms provide a first overview of utilized shapes within a building floor plan. Our visualization helps to investigate these shapes in different manners:

1) You can easily see the location of the individual shapes. Together with external information such as purpose or direction and the same information about neighboring shapes, you can draw conclusions about the design and use them in your own design.

2) With the help of the information about the number of different shapes that occur in the respective building, the architects can draw conclusions about the complexity of the building.

3) Due to the different colors of the individual shapes, groupings and tendencies are easy to recognize. In Figure 8, for example, you can see that the upper part of the floor plan is largely monotonous and simple, while the more complex structures are all located in the lower part.

This allows architects to identify shapes that are used widely in a building design or identify symmetries visually.

The computation of the presented algorithm can be achieved in mostly 5 seconds on an ordinary desktop computer. Still, the computational complexity is depending on the number of defined rooms. Right now, each identified room is analyzed 


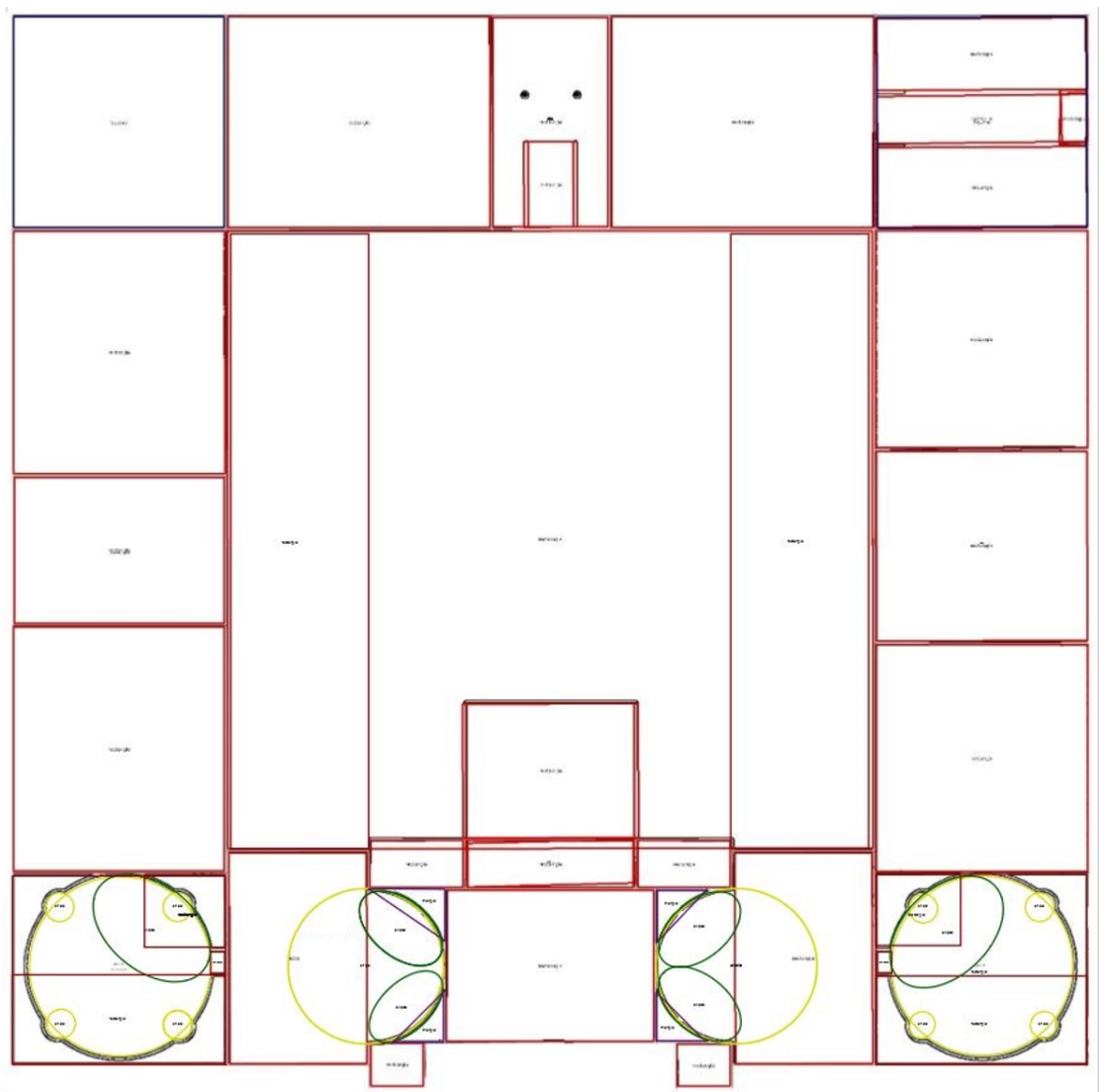

a)

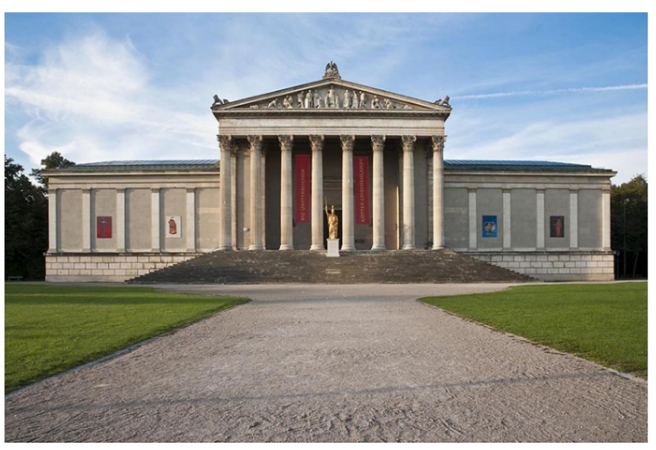

b)

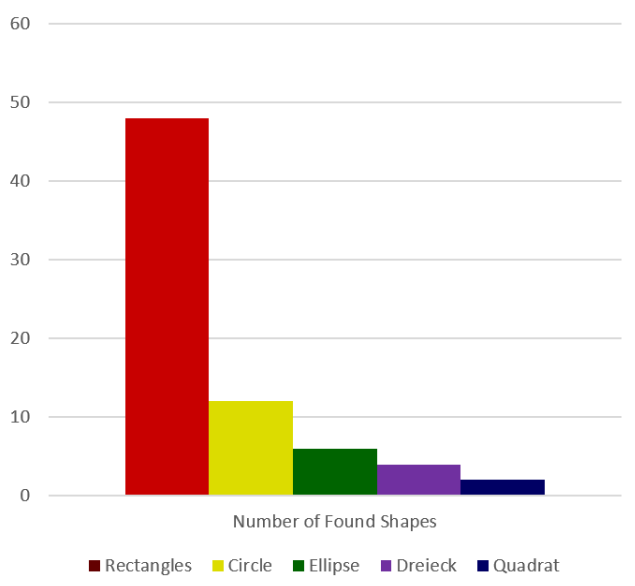

c)

Fig. 8. Our approach for shape analysis and visualization applied to a real world building floor plan of the Glyptothek in Munich [27] c). a) The identified shapes are drawn into the building floor plan and their number of occurrences is summarized in a barchart c).

one after another. As rooms are independent of each other, the computation can be performed parallel.

With the help of the display of how many and which geometric shapes are present in the building under consideration, the architect can analyze the preferences and tendencies of other architects or with regard to buildings that serve a specific purpose, such as schools or museums.

The results indicate that the potential of the analysis tool is far from being exhausted. As mentioned above the composition of the rooms also belongs to the pure form of the building. Our collaborators mentioned that the tool should be expanded to include the analysis of proportions, hierarchies and symmetry in a further version. The recognition of the geometric shapes is not sufficient, since this only reflects a fraction of the analysis tasks of the design process. For this reason, our collaboration partners demand the above-mentioned extensions to the tool, as they would greatly simplify and accelerate the analysis of floor plans necessary for the design process. However, the recognition of the geometric figures represents a first necessary step.

As the results indicate, rectangular shapes hold the potential to overfit. This means, that more rectangles might be identified, as they are present in the image. Besides, ellipses and circles are hard to distinguish. Parts of ellipses might be curved enough that they could be misinterpreted as circles or vice versa. Still, our collaborators rated our methodology as a good starting point from where they can examine building floor plans.

\section{CONCLUSION}

In this work we presented an automated tool for the shape detection and visualization in building floor plans. The method describes a pipeline that carefully selects preprocessing, shape detection, shape identification and visualization approaches to provide architects with a visual analysis of a building floor plan. We showed the effectiveness of the presented approach by applying it to a real world data set and evaluate the results together with our domain scientists.

As future work, we aim to provide an analysis tool that collects the retrieved shapes from multiple building plans and derives rules for best practices based on further parameters such as proportion rules, use of building or the architect.

\section{REFERENCES}

[1] Nizam Sönmez. Architectural layout evolution through similarity-based evaluation. International Journal of Architectural Computing, 13:271298, 122015 . 
[2] Jürgen Döllner and Henrik Buchholz. Continuous level-of-detail modeling of buildings in 3d city models. In Proceedings of the 13th Annual ACM International Workshop on Geographic Information Systems, GIS '05, page 173-181, New York, NY, USA, 2005. Association for Computing Machinery.

[3] Stephen Balaban. Deep learning and face recognition: the state of the art. page 94570B, 052015.

[4] Diep Thi Ngoc Nguyen. State-of-the-art in action: Unconstrained text detection. In Proceedings of the IEEE/CVF International Conference on Computer Vision (ICCV) Workshops, Oct 2019.

[5] Daniel Hansen, Kamal Nasrollahi, Christoffer Rasmusen, and Thomas Moeslund. Real-time barcode detection and classification using deep learning. pages 321-327, 012017.

[6] Y. Liu, Z. Zhang, R. Zhong, D. Chen, Y. Ke, J. Peethambaran, C. Chen, and L. Sun. Multilevel building detection framework in remote sensing images based on convolutional neural networks. IEEE Journal of Selected Topics in Applied Earth Observations and Remote Sensing, 11(10):3688-3700, 2018

[7] Tobias Post, Christina Gillmann, Thomas Wischgoll, and Hans Hagen. Fast 3D Thinning of Medical Image Data based on Local Neighborhood Lookups. In Enrico Bertini, Niklas Elmqvist, and Thomas Wischgoll, editors, EuroVis 2016 - Short Papers. The Eurographics Association, 2016.

[8] Sven Loncaric. A survey of shape analysis techniques. Pattern Recognit. 31(8):983-1001, 1998.

[9] Zhong-Qiu Zhao, Peng Zheng, Shou-tao Xu, and Xindong Wu. Object detection with deep learning: A review, 2018. cite arxiv:1807.05511.

[10] Zhengxia Zou, Zhenwei Shi, Yuhong Guo, and Jieping Ye. Object detection in 20 years: A survey. ArXiv, abs/1905.05055, 2019.

[11] Alexandru Costache, Dan Popescu, Loretta Ichim, and Ştefan Mocanu. Building recognition in static images. 2019 23rd International Conference on System Theory, Control and Computing (ICSTCC), pages 486-491, 2019.

[12] Matthias Zeppelzauer, Miroslav Despotovic, Muntaha Sakeena, David Koch, and Mario Döller. Automatic prediction of building age from photographs. CoRR, abs/1804.02205, 2018.

[13] Danny Lobos and Dirk Donath. The problem of space layout in architecture: A survey and reflections. Arquitetura Revista, 6:136-161, 122010.

[14] Samuel Dodge, Jiu Xu, and Bjorn Stenger. Parsing floor plan images. pages 358-361, 052017.

[15] Christina Gillmann, Pablo Arbelaez, Jose Tiberio Hernandez, Hans Hagen, and Thomas Wischgoll. An uncertainty-aware visual system for image pre-processing. Journal of Imaging, 4(9), 2018.

[16] Sébastien Macé, Hervé Locteau, Ernest Valveny, and Salvatore Tabbone. A system to detect rooms in architectural floor plan images. pages 167174, 062010

[17] Brian Okorn, Xuehan Xiong, and Burcu Akinci. Toward automated modeling of floor plans. 2010.

[18] Hanme Jang, Jong Hyeon Yang, and Yu Kiyun. Automatic Wall Detection and Building Topology and Property of 2D Floor Plan (Short Paper). In Stephan Winter, Amy Griffin, and Monika Sester, editors, 10th International Conference on Geographic Information Science (GIScience 2018), volume 114 of Leibniz International Proceedings in Informatics (LIPIcs), pages 33:1-33:5, Dagstuhl, Germany, 2018 Schloss Dagstuhl-Leibniz-Zentrum fuer Informatik.

[19] Zahra Ziran and Simone Marinai. Object Detection in Floor Plan Images, pages 383-394. 092018.

[20] Adrian Kaehler and Gary Bradski. Learning OpenCV 3: computer vision in $C++$ with the OpenCV library. "O'Reilly Media, Inc.", 2016.

[21] Herbert Süße and Erik Rodner. Bildverarbeitung und Objekterkennung. Springer, 2014.

[22] M. Balszun, M. Geier, and S. Chakraborty. Predictable vision for autonomous systems. In 2020 IEEE 23rd International Symposium on Real-Time Distributed Computing (ISORC), pages 116-123, 2020.

[23] Thanh Minh Nguyen, Siddhant Ahuja, and QM Jonathan Wu. A realtime ellipse detection based on edge grouping. In 2009 Ieee International Conference on Systems, Man and Cybernetics, pages 3280-3286. IEEE, 2009.

[24] Dilip K Prasad, Maylor KH Leung, and Siu-Yeung Cho. Edge curvature and convexity based ellipse detection method. Pattern Recognition, 45(9):3204-3221, 2012
[25] Andrew Fitzgibbon, Maurizio Pilu, and Robert B Fisher. Direct least square fitting of ellipses. IEEE Transactions on pattern analysis and machine intelligence, 21(5):476-480, 1999.

[26] Christina Gillmann, Heike Leitte, Thomas Wischgoll, and Hans Hagen From Theory to Usage: Requirements for successful Visualizations in Applications. In IEEE Visualization Conference (VIS) - C4PGV Workshop, 2016.

[27] Turbopass. Glyptothek und Staatl. Antikensammlungen. https://www.turbopass.de/muenchen/glyptothek.html, 2020. Added at: $2020-01-20$.

[28] Blogger. Architektur des Klassizismus. http://syndrome-destendhal.blogspot.com/2019/05/architektur-des-klassizismus-1-die.html, 2020. Added at: 2019-05-25. 